Bull. Austral. Math. Soc.

$20 \mathrm{~F} 29,20 \mathrm{~F} 38,20 \mathrm{c} 32$

Vol. 50 (1994) [1-11]

\title{
GROUPS WITH NO NONTRIVIAL LINEAR REPRESENTATIONS
}

\section{A.J. BERRICK}

We study the class of groups having no nontrivial linear representations over certain fields. After showing the class to be closed under perfect extensions with locally soluble kernel, we expand considerably the number of acyclic groups known to be in the class, by application to both binate groups and the acyclic automorphism groups of de la Harpe and McDuff.

Our aim is to initiate the study of groups $G$ whose finite-dimensional linear representations $G \rightarrow G L_{n}(\mathfrak{k})$ over a field $\mathfrak{k}$ are all necessarily trivial. This note has three main features:

(1) We survey the existing literature on such groups. Noteworthy here is the surprising interaction with the phenomenon of acyclic (homologically trivial) groups. For further discussion see Remark 1.10 below.

(2) We contribute to the theory of this class of groups by showing it to be closed under perfect extension with locally soluble kernel (Theorem 2.1).

(3) Applications of this key result are given to two important classes of acyclic groups, namely binate groups and groups of automorphisms of large objects (Theorems 3.1, 3.4).

We call a group $G$ with no nontrivial finite-dimensional k-representation counter$\mathfrak{k}$-linear. For $p \geqslant 0$, we say that $G$ is counter-p-linear if it is counter $\mathfrak{k}$-linear for every field $\mathfrak{k}$ of characteristic $p$. Finally, when $G$ is counter- $p$-linear for all $p$ (or equivalently, counter-k-linear for all $\mathfrak{k}$ ), we say that $G$ is counter-linear.

(Evidently we are considering a special case of the following. Let $\mathcal{C}$ be a class of groups. Then we may call a group $G$ counter $\mathcal{C}$ if $G$ has no nontrivial homomorphic image in $\mathcal{C}$. A brief discussion of this general notion appears below as an appendix.)

\section{EXAMPLES}

EXAMPLE 1.1. Perhaps chronologically the first example is Higman's four generator, four relator group

$$
H=\left\langle x_{i}, i \in \mathbb{Z} / 4 \mid\left[x_{i}, x_{i+1}\right]=x_{i+1}\right\rangle
$$

which is shown in [18] (by means of combinatorial group theory) to be counter-finite. Then, as in (2.5) below, it is also counter-linear. This also appears to be the first example in the literature of an acyclic group.

Received 16 August 1993

Copyright Clearance Centre, Inc. Serial-fee code: 0004-9729/94 \$A2.00+0.00. 
EXAMPLE 1.2. By a theorem of Zassenhaus [23, (15.1.3)] any perfect, locally soluble group is counter-linear. (Compare (2.4) below.) In particular, the perfect, locally nilpotent groups of McLain [23, (12.1.9)] are counter-linear, as are their generalisations in [3] which can have as their centre any given Abelian group. These groups are also acyclic [3]. Also, Heineken and Wilson [16] exhibit locally soluble groups with minimal condition on normal subgroups. The intersection of the derived series is therefore a perfect, locally soluble group.

EXAMPLE 1.3. The extreme subclass of counter-linear groups is the class of simple non-linear groups. A simple, locally finite group $G$ is, according to [19], counterlinear if for all primes $\boldsymbol{p}$ there is an infinite strictly descending chain of $\boldsymbol{p}$-subgroups, or equivalently, if $G$ involves all finite groups. For an example, see [14]. Further examples include certain existentially closed groups, which are again acyclic [4].

EXAMPLE 1.4. For any associative ring $R$ with 1 , consider the group $G=E R$ which is the union of all the groups $E_{m}(R)$ generated by elementary $m \times m$ matrices (nested by adjunction of identity matrices). Then $G$ contains, for example, elementary matrices of the form $I_{m}+r e_{1 m}$ where $r \in R$ is arbitrary. Note that these elementary matrices lie in the $k$ th term of the derived series of the upper unitriangular matrix group $U_{m}(R)$, where $k=\left[\log _{2}(m-1)\right]+1$ and so increases unboundedly with $m$. If now $G$ admits a finite-dimensional representation $\rho$, then by Zassenhaus it follows that there is a bound on the derived length of the image of any soluble subgroup of $G$ under the representation. Therefore for arbitrary $r \in R$ the matrix $I_{m}+r e_{1 m}$ lies in the kernel of $\rho$. However, the conjugates of such matrices generate $G$. This forces $\rho$ to be trivial. Hence $G$ is counter-linear.

When the Milnor $K$-group $K_{2} R$ of $R$ is nonzero, an interesting phenomenon occurs here. For $K_{2} R$ is just the Schur multiplier of $G$. By the relationship between Schur multipliers and projective representations [10, (II.15)], it follows that when the multiplier is nonzero there exists a projective representation $G \rightarrow P G L(V)$ of $G$ which fails to lift to a linear representation $G \rightarrow G L(V)$, where $V$ is a complex vector space. Now, as in Theorem 2.1 below, there is an embedding $P G L(V) \hookrightarrow G L(R(P G L(V)))$. Thus counter-linearity forces any finite-dimensional projective representation of $G$ to be trivial and so equivalent to a linear representation. On the other hand, because $K_{2} R \neq 0$, there exists an infinite-dimensional such $V$, and thereby a nontrivial infinitedimensional linear representation $G \rightarrow P G L(V) \hookrightarrow G L(R(P G L(V)))$.

EXAMPLE 1.5. Let $D$ be a noncommutative division algebra which is infinite-dimensional over its centre. Then by [11] the commutator subgroup $S L_{n}(D)$ of $G L_{n}(D)$ is counterlinear $(n \geqslant 2)$, as is the special unitary group $S U_{n}(D)$ with Witt index greater than 1. Proofs here use the theory of algebraic groups. 
EXAMPLE 1.6. By topological methods, [5] shows that any torsion-generated acyclic group is counter- $\mathbb{C}$-linear. (Recall that such a group is generated by elements of finite order and has the same homology, with trivial coefficients, as the trivial group.) This paper also makes a number of deductions about the internal structure of such groups which are applicable more generally to perfect, counter-finite or counter- $\mathbb{C}$-linear groups. For example, if a normal subgroup $N$ has $N / N^{\prime \prime}$ finitely generated, then $N$ is either finitely generated Abelian or (infinite perfect)-by-(finitely generated Abelian). A further example occurs in (2.6) below.

EXAMPLE 1.7. Again using algebraic topology, [7] generalises the above results, proving the counter- $\mathbb{C}$-linearity of any torsion-generated group $G$ having only finitely many nonzero (trivial coefficient) homology groups $H_{n}(G ; \mathbb{Z})$. Note here that the Schur multiplier $\mathrm{H}_{2}(G ; \mathbb{Z})$ need not vanish; indeed, according to [7] any Abelian group is possible for $\mathrm{H}_{2}(G ; \mathbb{Z})$. Then the argument concerning projective representations given in Example 1.4 also applies here, at least with respect to complex vector spaces $V$.

EXAMPLE 1.8. Another interesting example from [7] (settling an issue raised in [2]) is of a (universal) finitely presented torsion-generated acyclic group. From Example 1.5 and (2.5) below, it follows that this group must be counter-linear. On the other hand, [2] exhibts a finitely presented torsion-free acyclic group which has the simple group of order 60 as a quotient.

EXAMPLE 1.9. Strongly torsion generated groups were introduced in [6] and analysed in [7]. Their characteristic property is that, for each $n \geqslant 2$, there is an element $x_{n}$ of order $n$ whose conjugates generate the group $G$. Exidently any homomorphic image is also strongly torsion generated, so we consider the case when $G$ is linear. Then over characteristic $p>0$ we have

$$
\left(x_{p}-1\right)^{p}=x_{p}^{p}-1=0 ;
$$

this makes $x_{p}$ and its conjugates unipotent. Since the conjugates of $x_{p}$ generate $G$, $G$ is itself unipotent, hence nilpotent. However, the only Abelian strongly torsion generated group is trivial. We conclude that strongly torsion generated groups are counter-p-linear for all $p>0$.

REMARK 1.10. In the the above examples, certain aspects of the relation between counter-linearity and acyclicity are worth emphasising. General implications of counterlinearity from acyclicity, as in (1.6) - (1.9), rely on the presence of torsion. For torsionfree acyclic groups, more ad hoc methods have had to be used, as in (1.1), (1.2). Clearly it would be desirable to find a more general approach to counter-linearity for acyclic groups. That this is impossible in complete generality follows from examples of torsionfree acyclic groups with nontrivial finite quotients [2]. In fact, given any perfect group 
$P$, it is known that there is a torsion-free acyclic group with $P$ as quotient [17]. A large class of acyclic groups, including many without torsion, arises via binate groups. This prompts investigation of the counter-linearity of binate groups, aided by the work of [9] on binate towers. A partial result concerning non-linearity of the universal binate tower has been obtained in [12]; it motivated the present work.

We first obtain a key lemma on counter-linearity and extensions. When combined with work of [1] on the universal binate tower it leads to counter-linearity of binate and related acyclic groups.

\section{BASIC RESULTS}

By means of the following fundamental result, we are able to enlarge considerably the class of counter- $k$-linear groups. Throughout $k$ always denotes a field whose algebraic closure is $\overline{\mathrm{E}}$.

TheOREM 2.1. There is a function $m=m(n)$ with the following property. Suppose that a group $G$ has a nontrivial $n$-dimensional k-representation. Then either

(i) $G$ has a nontrivial 1-dimensional $\overline{\mathrm{k}}$-representation, or

(ii) for each locally soluble proper normal subgroup $N$ of $G$ there is a nontrivial m-dimensional k-representation of $G / N$.

Proof: By passing to the image of the given representation, we may assume $G$ to be a subgroup of $G L_{n}(\mathfrak{k})$. Then by Zassenhaus [23, (15.1.3)] $N$ is soluble, of derived length $d$ bounded by a function $d(n)$ of $n$. Since one can argue inductively up the derived series of $N$, it suffices to consider the case where $N$ is Abelian. Now for $K$ normal in $G$ we define $R(K)$ to be the k-subalgebra of $M_{n}(\mathfrak{k})$ generated by $K$. Then $G$ acts on $R(K)$, a subspace of $\mathrm{k}^{n^{2}}$, with kernel $C_{G}(K)$. Thus $G$ acts on $S=R(N) \oplus R\left(C_{G}(N)\right)$ with kernel $C_{G}(N) \cap C_{G}\left(C_{G}(N)\right)=\mathcal{Z}\left(C_{G}(N)\right) \geqslant N$, affording a k-representation of $G / N$. If this representation is trivial, then, because its Abelian kernel $\mathcal{Z}\left(C_{G}(N)\right) / N$ coincides with the group $G / N, G$ is Abelian $k$-linear and so is a subgroup of $G L_{1}(\mathfrak{B}) \times \ldots \times G L_{1}(\mathfrak{B})$. Evidently $m=m(n, d)=m(n, d(n))$; we may take $m(n, 0)=n$ and, for $d \geqslant 1, m(n, d)=2 m(n, d-1)^{2}$.

When $G$ is locally soluble we may take $N$ in the above to be the commutator subgroup $G^{\prime}$ of $G$. (It follows from the theorem of Zassenhaus cited above that $G^{\prime}$ must be proper in $G$ since $G$ has a nontrivial soluble image.) The complete reducibility of Abelian subgroups of $G L_{m}(\mathfrak{E})$ is now seen to have the following counterpart for locally soluble groups.

COROLlaRY 2.2. If a locally soluble group has a nontrivial k-representation of any dimension then it has a nontrivial 1-dimensional $\overline{\mathfrak{E}}$-representation. 
It is perhaps interesting to observe that $[21,(13.6)]$ shows that, in the special case of the above where the group algebra $\mathfrak{k}[G]$ has a faithful representation over some commutative $\mathfrak{k}$-algebra, then $G$ is locally soluble-by-finite.

Returning to counter-p-linear groups, we note that, under favourable circumstances, one can specialise to particular fields $\mathfrak{k}$. For this result, we recall that a rational group is one which embeds in $\mathbb{Q}$; and for prime $p \geqslant 0$ we write $\overline{\mathbb{F}}_{p}$ for the algebraic closure of the prime field of characteristic $p$.

\section{Theorem 2.3. Let $G$ have no noncyclic free subgroup.}

(a) For $p \geqslant 0, G$ is counter-p-linear if and only if $G$ is both counter- $\overline{\mathbb{F}}_{p}$-linear and counter-rational.

(b) The following are equivalent:

(i) $G$ is counter-0-linear;

(ii) $G$ is perfect and there exists a field $k$ of characteristic 0 with $G$ counter-k-linear;

(iii) $G$ is perfect and counter-finite.

Proof: (a) Note that the condition of counter-rationality has force only when $p>0$, since $\mathbb{Q}$ embeds in $G L_{2}(\mathbb{Q})$ (via unitriangular matrices). Now the "only if" direction is immediate, except that, when $p>0$, one also appeals to $(\alpha)$ (i) of the following data obtained from $[13,(127.3)]$ concerning the groups $G L_{1}(\mathfrak{k})$.

(a) Fix $p>0$. Then

(i) there exists a field $\mathfrak{k}$ with characteristic $p$ having $\mathbb{Q}$ a subgroup of $G L_{1}(k)$; and

(ii) for any field of characteristic $p$, a subgroup of $G L_{1}(\mathfrak{k})$ is counter-rational if and only if it is a subgroup of $G L_{1}\left(\mathbb{F}_{p}\right)$.

( $\beta$ ) Any nontrivial subgroup of $G L_{1}(\mathfrak{k})$ (with $\mathfrak{k}$ of characteristic 0 ) has a nontrivial quotient in $G L_{1}\left(\mathbb{F}_{0}\right)$.

For the converse argument, since the various properties are preserved by quotients, assume that $G \leqslant G L_{n}(\mathfrak{k})$ with $\operatorname{char} \mathfrak{k}=p$, and that $G$ is counter- $\overline{\mathbb{F}}_{p}$-linear and counterrational. By a result of Tits [25, pp.145,146] this forces $G$ to have a soluble normal subgroup $N$ with $G / N$ locally finite. If $G$ is itself soluble, then by the corollary above $G$ has a nontrivial image in $G L_{1}(\mathfrak{E})$. Similarly if $G$ is not soluble (so that $N$ is proper) and (1.1)(i) applies. Therefore, by $(\alpha)(i i)$ and $(\beta)$ above, $G$ has a nontrivial image in $G L_{1}\left(\bar{F}_{p}\right)$. This leaves the case where we may apply $(2.1)(i i)$ to the pair $G, N$. Here we obtain a nontrivial $\bar{k}$-representation of locally finite $G / N$. Then the argument of $[20,(1 . L .2)]$ leads to a nontrivial $\bar{F}_{p}$-representation, as required.

(b) To see that (i) $\Rightarrow$ (ii), note that, because $G L_{1}(\mathbb{C})$ is divisible (hence injective) and contains all possible torsion, any Abelian group has a nontrivial image in $G L_{1}(\mathbb{C})$. 
Thus, counter- $\mathbb{C}$-linear implies perfect.

Next, (ii) $\Rightarrow$ (iii) because every finite group is $\mathbb{Q}$-linear. There remains the proof that (iii) $\Rightarrow$ (i). As with (a), from a nontrivial k-representation $\rho: G \rightarrow G L_{n}(\mathfrak{k})$ we obtain a nontrivial locally finite image $Q$ of $G$ which is k-linear. (Since $G$ is perfect the other possibilities are excluded.) However, since $k$ has characteristic zero, a result of Schur [20, (1.L.4)] gives $Q$ as Abelian-by-finite. Because $G$ is perfect, this forces $Q$, hence $G$, to have a nontrivial finite image.

Combination of the last two results readily yields the following.

Corollary 2.4. Let $G$ be a locally soluble group. Then the following statements are equivalent:

(i) $G$ is counter-linear;

(ii) $G$ is counter- $\overline{-}-$-linear for some field $\mathfrak{k}$ of characteristic 0 ;

(iii) $G$ is perfect.

Although well-known, the following is worth noting in this context.

Proposition 2.5. Suppose $G$ is finitely generated. Then the following are equivalent.

(i) $G$ is counter-linear.

(ii) There exists a field $\mathfrak{k}$ with $G$ counter-k-linear.

(iii) $G$ is counter-finite.

Proof: Evidently (i) $\Rightarrow$ (ii), while (ii) $\Rightarrow$ (iii) because any finite group has a faithful representation by permutation matrices. Finally, after Mal'cev [25, p.51], finitely generated linear groups are known to be residually finite; thus (iii) $\Rightarrow$ (i).

The next result belongs to the genre of [5]. Here we apply it to obtain further information about perfect group extensions with counter-linear quotient.

Lemma 2.6. If a soluble minimax subgroup $N$ of a counter-0-linear group $G$ is normal in $G$, then it is central.

Proof: Since $G$ is perfect (compare (2.3)(b)) we pass to the centreless group $G / \mathcal{Z}(G)$, or equally, assume $G$ to be centreless. Again, by considering the minimal nontrivial term of the derived series of $N$, we may assume $N$ to be Abelian. Now the torsion subgroup $T(N)$ of $N$ has $\min [23$, (Exercise 4.4.7)] and so is Cernikov. By [20, (3.38)] it follows that its automorphism group is linear over some field of characteristic zero. Thus conjugation by $G$ on $T(N)$ is trivial; since $G$ is centreless, this makes $N$ torsion-free. Various standard results on torsion-free Abelian minimax groups [23, Chapter 4] imply that $N$ has finite rank, $m$ say, and embeds as a subgroup of a rational vector space of dimension $m$. By tensoring over $\mathbb{Q}$ any automorphism of $N$, we obtain 
an embedding of Aut $(N)$ in $G L_{m}(\mathbb{Q})$. Again this forces the conjugation action of $G$ on $N$ to be trivial, whence $N$ is trivial as required.

THEOREM 2.7. Let $N$ be a soluble minimax group. Then $N$ can be the kernel of a perfect group extension with counter-0-linear quotient if and only if $N$ is Abelian and the extension is central.

Proof: First suppose that the extension is not central. Then by the lemma the extension group cannot be counter- 0 -linear. Then Theorem 2.1 implies that the quotient group also fails to be counter-0-linear. On the other hand, if $N$ is Abelian then as in (1.2) above it follows from [3] that $N$ embeds as the centre of a perfect counter-linear group.

Note that in (2.7) the hypothesis of perfectness is necessary, to avoid for example the possibility that the extension reduces to a direct product of $N$ with the quntient group.

\section{Applications}

We now apply Theorem 2.1 to results of [1] to discover new classes of counter-0linear groups. Recall from [4] that binate groups form a very important class of acyclic groups. A binate group $G$ has the property that for each finitely generated (necessarily proper) subgroup $H$ of $G$ there is a homomorphism $\varphi: H \rightarrow G$ and element $a \in G-H$ such that for all $h \in \boldsymbol{B}$

$$
h=[\varphi(h), a] .
$$

Since the subgroup generated by $H, \varphi(H)$ and $a$ is in turn finitely generated, this allows one to iterate, leading to a binate tower of finitely generated subgroups $H=$ $H_{0} \leqslant H_{1} \leqslant \ldots$. Binate towers are analysed at length in [9].

THEOREM 3.1. Any binate group $G$ is counter-0-linear.

Proof: Suppose otherwise, and let $G$ have a nontrivial representation $\rho: G \rightarrow$ $G L_{n}(\mathfrak{k})$, where $\mathfrak{k}$ has characteristic zero. Given a finitely generated subgroup $H$ of $G$, let $H=H_{0} \leqslant H_{1} \leqslant \ldots$ be a binate tower in $G$ with base $H$. Then, by $[1,(1.1)]$, each $\rho\left(H_{i}\right)$ is soluble-by-finite. This makes $\rho(G)$ (locally soluble)-by-(locally finite). So we obtain from Theorem 2.1 above a nontrivial representation $\nu: \rho(G) \rightarrow G L_{m}(\mathfrak{k})$ with $\nu \rho(G)$ locally finite. However, by a result of Schur [20, (1.L.4)], $\nu \rho(G)$ has an Abelian subgroup of finite index. Since $\nu \rho(G)$ is perfect, the subgroup must be proper. Hence $G$ has a proper subgroup of finite index, contradicting $[1,(1.4)(\mathrm{d})]$.

Recall from [4] that any group is normal in a normal subgroup of a binate group. We therefore have an immediate consequence. 
Corollary 3.2. Any group is two-step subnormal in a counter-0-linear group.

However, Lemma 2.6 above shows that there are considerable constraints on the class of normal subgroups of counter- 0 -linear groups.

These techniques also enable one to say something about positive characteristics.

Corollary 3.3. If a binate group $G$ has no proper normal subgroup of countable index, then $G$ is counter-linear.

ProOf: Again suppose the existence of a nontrivial representation $\rho: G \rightarrow$ $G L_{n}(\mathfrak{k})$, where $\mathfrak{k}$ is now any field. As above, $G$ has a nontrivial locally finite image in $G L_{m}(\mathfrak{k})$ for some $m$. Then the result follows from [27], [20, (1.L.2)].

The above result does not apply directly to the acyclic groups of automorphisms considered by de la Harpe and McDuff [15] (although they are known to be countercountable), for they need not be binate. Recall that those groups are the following:-

(a) the group of all permutations of any infinite set;

(b) the group of measure-preserving automorphisms of a Lebesgue measure space with infinite, nonatomic measure;

(c) the group of all linear automorphisms of an infinite-dimensional vector space;

(d) the group of all continuous linear automorphisms of an infinite-dimensional Hilbert space $V$ over $\mathbb{R}, \mathbb{C}$ or $\mathbb{H}$, as well as the group of invertible isometries of $V$;

(e) the group of invertible elements in a properly infinite von Neumann algebra, and its subgroup of unitary elements.

However, the proof of acyclicity of the above groups relies heavily on the existence of families of binate subgroups. Thus, with a little care, one can modify our previous argument for binate groups, to obtain the following result.

THEOREM 3.4. The groups of [15] are counter-linear.

Proof: Let $G$ be such a group, with nontrivial representation $\rho: G \rightarrow G L_{n}(\mathfrak{k})$. For each flag $F$ defined in the grassmannian of subspaces of the space on which $G$ acts, there is a subgroup $G_{F}$ of $G$ defined in [15]. By applying the fundamental group functor to Lemma 11 of [15] (or, more directly, from Lemma 10 of [15] - see also (3.3) of [24]), one sees that $G$ is the union of finite products of subgroups of the form $G_{F}$. Moreover, each $G_{F}$ is a semi-direct product $A \rtimes G_{F}^{\prime}$ of an Abelian normal subgroup $A$ by a binate group $G_{F}^{\prime}$ (see [15, Section 2] and [4, (3.8)]). Now by $[1,(1.1)] \rho\left(G_{F}^{\prime}\right)$ is (locally soluble)-by-(locally finite), and hence, by Zassenhaus [23, (15.1.3)] soluble-by-(locally finite). Thus each $\rho\left(G_{F}\right)$ is also soluble-by-(locally finite), and again, after taking the direct limit of finite products, we have that the perfect group $\rho(G)$ is soluble-by-(nontrivial locally finite). So, by $(2.1)$ above, $\rho(G)$ has a nontrivial 
locally finite $k$-linear image, and hence, by [20, (1.L.2)] a nontrivial countable image. However, this contradicts Corollary A4 of [15].

Remark 3.5. Subsequent work [1, Section 3] shows that it is possible to remove the condition on the binate group $G$ in (3.3). Using this result, I believe one can construct an alternative proof of (3.4) above.

\section{APPENDIX ON COUNTER- $\mathcal{C}$ GROUPS}

Let $\mathcal{C}$ be a class of groups. We call a group $G$ counter- $\mathcal{C}$ (or say that $G$ belongs to the class counter- $\mathcal{C}$, denoted $\mathcal{C}^{-H}$ in [22]) if $G$ has no nontrivial homomorphic image in $\mathcal{C}$. One seeks to describe the class of all counter- $\mathcal{C}$ groups. Some cases of particular interest are the following.

The counter-finite groups are those with no proper subgroups of finite index. (I understand that an investigation of (counter) ${ }^{2}$-finite groups is now being made by V. Walter, a student of D.J.S. Robinson at Urbana.) The counter-Abelian (equally, counter-nilpotent, counter-soluble) groups are just the perfect groups. One can iterate the process: the (counter) ${ }^{2}$-Abelian groups are the imperfect groups; these form the object of study in [8]. Then the (counter) ${ }^{3}$-Abelian groups are the perfect groups again (for, their commutator quotient groups must be Abelian groups with no nontrivial Abelian image, hence trivial). Thus in this case the iteration becomes stationary. In fact, in general at most three new classes of groups may be produced by iteration in this way, as is seen from the following chain of easily checked results. (For an alternative derivation of (v) from (i), see [22] (1.39).)

Proposition A.1. Let $\mathcal{C}$ be a class of groups. Then

(i) the class counter- $\mathcal{C}$ is closed under the formation of quotients;

(ii) $\mathcal{C} \subseteq$ (counter) $)^{2} \mathcal{C}$ if and only if $\mathcal{C}$ is closed under the formation of simple quotients;

(iii) counter $-\mathcal{C} \subseteq$ (counter) $)^{3}-\mathcal{C}$;

(iv) (counter) $)^{3}-\mathcal{C}=$ counter- $\mathcal{C}$ if and only if $\mathcal{C}$ is closed under the formation of simple quotients;

(v) $(\text { counter })^{4}-\mathcal{C}=(\text { counter })^{2}-\mathcal{C}$.

The concept of counter- $\mathcal{C}$ groups is in a sense dual to that of just-not- $\mathcal{C}$ groups (whereby every homomorphic image, but not the group itself, lies in $\mathcal{C}$ ) studied by Wilson [26] et al.

\section{REFERENCES}

[1] R.C. Alperin and A.J. Berrick, 'Linear representations of binate groups', J. Pure Appl. Algebra (to appear). 
[2] G. Baumslag, E. Dyer and A. Heller, 'The topology of discrete groups', J. Pure Appl. Algebra 16 (1980), 1-47.

[3] A.J. Berrick, 'Two functors from abelian groups to perfect groups', J. Pure Appl Algebra 44 (1987), 35-43.

[4] A.J. Berrick, 'Universal groups, binate groups and acyclicity', in Group Theory, Proceedings of the Singapore Conference (Walter de Gruyter, Berlin, 1989), pp. 253-266.

[5] A.J. Berrick, 'Remarks on the structure of acyclic groups', Bull. London Math. Soc. 22 (1990), 227-232.

[6] A.J. Berrick, 'Torsion generators for all abelian groups', J. Algebra 139 (1991), 190-194.

[7] A.J. Berrick and C.F. Miller III, 'Strongly torsion generated groups', Math. Proc. Cambridge Philos. Soc. 111 (1992), 219-229.

[8] A.J. Berrick and D.J.S. Robinson, 'Imperfect groups', J. Pure Appl. Algebra 88 (1993), 3-22.

[9] A.J. Berrick and K. Varadarajan, 'Binate towers of groups', Arch. Math. 62 (1994), 97-111.

[10] F.R. Beyl and J. Tappe, Group extensions, representations, and the Schur multiplier, Lecture Notes in Math. 958 (Springer-Verlag, Berlin, Heidelberg, New York, 1982).

[11] Y. Chen, 'On the representations of skew linear groups', Bull. London Math. Soc. 21 (1989), 267-269.

[12] E. Formanek and C. Procesi, 'The automorphism group of a free group is not linear', $J$. Algebra 149 (1992), 494-499.

[13] L. Fuchs, Infinite Abelian groups II (Academic Press, New York, 1973).

[14] J.I. Hall and B. Hartley, 'A group theoretical characterization of simple, locally finite, finitary linear groups', Arch. Math. 60 (1993), 108-114.

[15] P. de la Harpe and D. McDuff, 'Acyclic groups of automorphisms', Comment. Math. Helv. 58 (1983), 48-71.

[16] H. Heineken and J.S. Wilson, 'Locally soluble groups with min-n', J. Austral. Math. Soc. 17 (1974), 305-318.

[17] A. Heller, 'On the homotopy theory of topogenic groups and groupoids', Mlinois J. Math. 24 (1980), 576-605.

[18] G. Higman, 'A finitely generated infinite simple group', J. London Math. Soc. 26 (1951), 61-64.

[19] O.H. Kegel, 'Über einfache, lokal endliche Gruppen', Math.Z. 95 (1967), 169-195.

[20] O.H. Kegel and B.A.F. Wehrfritz, Locally finite groups, North-Holland Math. Library 3 (North-Holland, Amsterdam, 1973).

[21] D.S. Passman, Infinite group rings, Pure and Appl. Monographs 6 (Marcel Dekker, New York, 1971).

[22] D.J.S. Robinson, Finiteness conditions and generalized soluble groups I (Springer-Verlag, Berlin, Heidelberg, New York, 1972).

[23] D.J.S. Robinson, $A$ course in the theory of groups, Graduate Texts in Mathematics 80 (Springer-Verlag, Berlin, Heidelberg, New York, 1982). 
[24] G. Segal, 'Classifying spaces related to foliations', Topology 17 (1978), 367-382.

[25] B.A.F. Wehrfritz, Infinite linear groups (Springer-Verlag, Berlin, Heidelberg, New York, 1973).

[26] J.S. Wilson, 'Groups with every proper quotient finite', Math. Proc. Cambridge Philos. Soc. 69 (1971), 373-390.

[27] D.J. Winter, 'Representations of locally finite groups', Bull. Amer. Math. Soc. 74 (1968), 145-148.

Department of Mathematics

National University of Singapore

Lower Kent Ridge Rd

Singapore 0511

Republic of Singapore

e-mail: matberic@nusunix.nus.sg 